\title{
Reduction des risques par les produits "prouvés moins nocifs»?
}

\section{Dr Rainer M. Kaelin}

Spécialiste en Medecine interne et Pneumologie, Membre FMH

Le parlement a retourné la loi sur les produits du tabac (LPTab) au conseil fédéral avec le mandat d'y ancrer la protection de la jeunesse, de supprimer l'interdiction de la publicité et d'y transférer de la loi des aliments uniquement l'essentiel concernant le tabac. Le ministre de la santé se trouve ainsi devant une mission impossible: Protéger la jeunesse sans interdiction globale de la publicité n'est pas réaliste. L'interdiction de vente aux mineurs est incontestée; mais le status juridique de la cigarette électronique est indéfini. Le conseiller national Pezzatti était de l'avis que «les produits prouvés moins nocifs» [1] devraient être soumis à des règles moins contraignantes.

Depuis que le Prof. Etter présenta la cigarette électronique comme une concurrente sur le marché du tabac $[2,3]$ la réduction des risques (harm reduction) est discutée. Ainsi certains médecins, des politiciens et les promoteurs défendent la consommation de nicotine sans combustion et/ou sans tabac. Cet article décrit l'origine de ce concept et sa place dans la prévention.

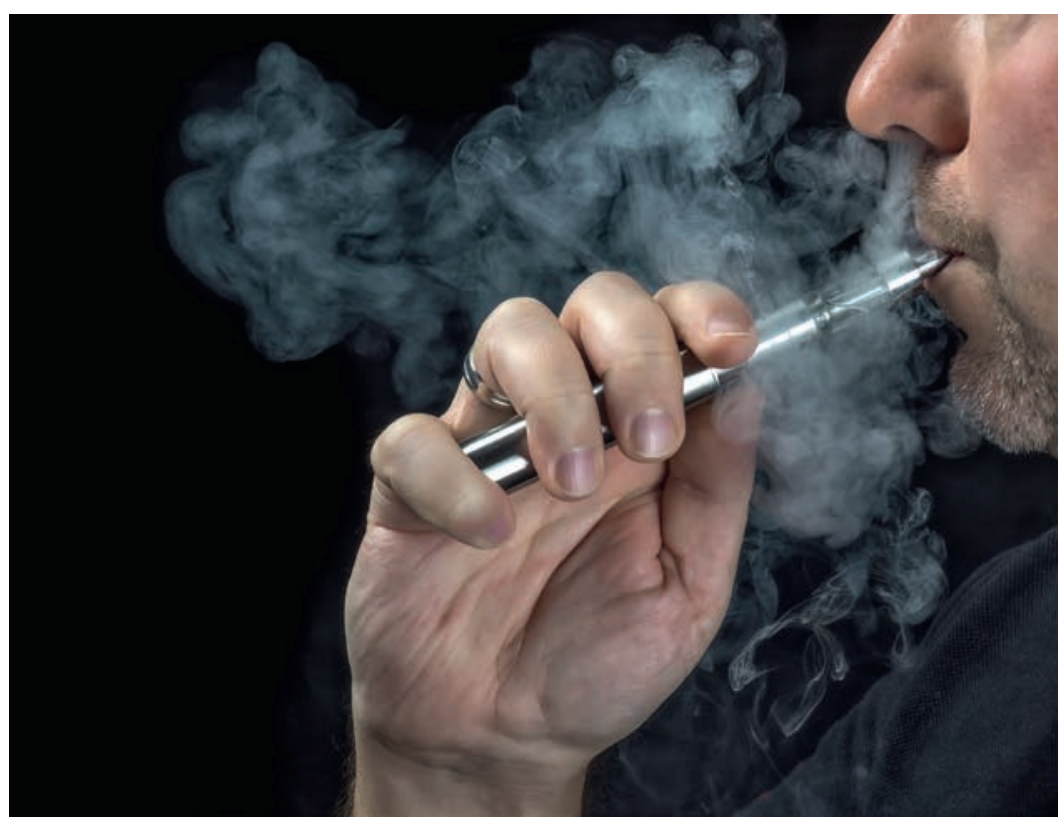

Vapoter entraîne une dépendance à la nicotine

\section{Additifs. La cigarette avec filtre}

La nicotine a été reconnue tôt comme essentielle pour capturer les fumeurs. La première cigarette qui, en 1913, contenait du tabac "Burley», plus riche en nicotine, était la Camel. Pour adoucir son âcreté, elle fut traitée par du sucre et de la réglisse, ce qui calmait la toux et permettait son inhalation [4], Lucky Strike (1916) et Chesterfield (1918) furent conditionnées de la même manière. Depuis, des apaisements comme "pas de toux", «nous avons enlevé du tabac les substances nocives et corrosives» [5], et des conseils médicaux firent partie de la publicité. Le traitement du tabac et le marketing visaient à camoufler l'irritation des voies aériennes causée par la nicotine et la fumée.

En 1964 le rapport du Surgeon general [6] concluait que la cigarette contribuait fortement à la mortalité des fumeurs. Les données d'alors étaient insuffisantes pour associer les cigarettes avec filtre à une diminution du risque. Néanmoins, celles-ci devinrent la norme dans les années 60. Toutefois la publicité ne quantifia jamais leur avantage sanitaire

\section{La cigarette légère. Dépendance de la nicotine}

En 1966 le contenu en goudron et en nicotine était examiné: «Les données suggèrent: plus les contenus en goudron et Nicotine sont bas, moins sont les dégâts» [7]. Ainsi la publicité sur le taux de goudron et de nicotine suggérait dans les années 70 une moindre nocivité, sans que des données fiables ne soient disponibles. Celles-ci furent obtenues par des machines fumer, mais c'est seulement en 1996 que la federal trade commission exigeait des indications sur ces taux, mesurées exclusivement par la méthode standardisée. Les "cigarettes légères» suggéraient un avantage non quantifié, malgré le fait qu'en 1981 déjà, un rapport du Surgeon General [8] avait conclu qu'il «n'existe pas de cigarette sûre (safe), ni de degré de consommation sans risque». Fumer des cigarettes au contenu de goudron moindre, pourrait diminuer le risque de cancer pulmonaire, mais «les bénéfices sont minimaux, par rapport 
à l'arrêt complet». Ce rapport confirmait que les indications sur les emballages sous-estimaient les doses reçues par le fumeur.

Les fabricants optimisaient l'absorption de nicotine par l'adjonction d'ammoniaque et de menthol. Ce dernier fut ajouté depuis les années 20 , afin de réduire l'irritation de la fumée et pour permettre de fumer, «même lors d'un refroidissement». Le Menthol est un anesthésiant qui supprime la toux et permet une inhalation profonde. Une faible dose (0,01-0,03\%) est ajouté même aux cigarettes non "mentholées», alors que celles-ci contiennent plus que $0,45 \%$ de leur poidstabac [9]. Elles sont populaires chez les jeunes et les fumeurs de ces cigarettes peinent à cesser leur tabagisme. Tardivement, en 2013, la FDA a été appelée à réglementer le menthol [10].

L'ammoniaque est ajoutée, car la nicotine par une fumée basique arrive plus rapidement (en 7-10 sec.) au cerveau et rend plus facilement dépendant. Elle est volatile, facilement absorbée en milieu basique, alors qu'en milieu acide, la nicotine prend une forme cristalline. Une des premières cigarettes à l'ammoniaque était la Marlboro (depuis 1956 fabriquée en dehors des USA à Neuchâtel) dont le succès commercial confirmait la meilleure absorption. La forme volatile n'est pas détectée par la machine à fumer [11], contrairement à sa forme liquide ou cristalline.

Les fabricants avaient compris la dépendance à la nicotine, base de l'épidémie mondiale du tabac, plus tôt que les professionnels de santé. C'est en 1988 que le Surgeon General l'évalua [12]. Le fumeur dépendant cherche d'un côté à atteindre par une montée nicotinique rapide sa «sensation de récompense», et, d'autre part, à éviter le manque, lorsque le taux de nicotine baisse après une pause [13]. C'est pourquoi les cigarettes légères ne signifient pas moins de fumée dans les alvéoles: puisque le fumeur cherche à maintenir un taux de nicotine plus ou moins constant, il tire plus sur sa cigarette, inhale plus souvent et fume plus proche du filtre. Les cigarettes «légères» créent ainsi des taux sanguins de monoxyde de carbone plus élevés.

\section{La convention cadre de l'OMS. L'e-cigarette}

Les produits affichés comme moins nocifs (potentially reduced exposure products, PREPS) des années 90 furent les précurseurs des cigarettes électroniques. Leur promotion "all of the taste... less of the toxins», "reduced carcinogens. Premium taste» intégrait la carcinogenicité que ces nouveaux produits éviteraient. Un comité mandaté par la FDA concluait en 2001 qu'il serait faisable de diminuer la carcinogenicité par réduction des produits toxiques issues du tabac [14] et employait pour ceci le terme "Harm reduction». Toutefois le rapport exprimait le soucis que les PREPS seraient utilisés par des fumeurs qui sans cela cesseraient de fumer; d'autres commenceraient à fumer percevant ces produits comme sûr (safe). Un quart de siècle après le rapport de 1981 concluant qu'il n'existe pas de cigarette «sûre», enfin on tenait compte, en 2006 de la perception erronée par le publique d'un risque réduit avec l'interdiction des termes «léger» et "contenu en Nicotine moindre».

Depuis le rapport de 1964, la cause de l'épidémie tabagique mondiale est identifiée dans le tabac et la nicotine, ainsi que dans l'action de l'industrie. C'est ce qui conduisit en 2004 à la convention cadre de l'OMS (CCLAT). Dans ce traité endossé par l'ONU, les pays retiennent que les produits du tabac sont développés pour créer une dépendance et que l'action néfaste de l'industrie doit être prise en compte pour endiguer le tabagisme mondial. L'article 5.3 de la CCLAT oblige les partenaires à protéger les mesures de prévention de leur sabordage par l'industrie. Entre santé publique et industrie un front était clairement défini.

L'e-cigarette introduite aux USA en 2005, brisa ce front. Gêné par aucun obstacle promotionnel, son marché se développa de manière fulgurante. Après une croissance annuelle de $24 \%$, Forbes indique 2,4 Mio de consommateurs en 2015 dans le Royaume-Uni [15] et trois fois plus aux USA , 43\% du marché mondial [16]. En Suisse, l'e-cigarette s'est répandue; en 2015 le nombre de ses utilisateurs d'au moins une fois a doublé en deux ans [17].

\section{Publicité et santé publique}

La cigarette électronique est dangereuse, car elle rend facilement accessible la nicotine bon marché. Elle est pour les jeunes une drogue d'entrée dans le tabagisme et vers d'autres dépendances $[18,19]$. L'aérosol de l'e-cigarette contient des carcinogènes, des particules fines, des métaux, et une multitude d'autres substances chauffées, dont l'inhalation répétée durant des années a des répercussions inconnues sur l'individu et la société.

Pour les adultes, sa promotion utilise le concept «réduction des risques» en faisant miroiter l'aide à l'arrêt du tabagisme et le remplacement de la cigarette tabac. Deux aspects très divergents sont mélangés. D’une part, l'e-cigarette pourrait aider certains fumeurs motivés à se libérer de leur dépendance; d'autre part le Prof. Etter défend pour la santé publique sa vision [1, 2]: puisque les fumeurs s'orientent vers la cigarette électronique, celle-ci, par le jeu du marché, va 
remplacer la cigarette tabac. Ceci n'est pas plausible. L' industrie du tabac, qui domine le marché, ne va pas promouvoir le nouveau engin par une stratégie «réduction des risques» efficace, ce qui reviendrait à saboter son premier marché, celui du tabac. L'utilisation fréquente et simultanée de la cigarette tabac et de la cigarette électronique indique qu'en général les fumeurs dépendants essaient (sans succès) de se nuire moins en «vapotant», mais ne veulent/peuvent pas abandonner le tabac.

Pour les jeunes, la promotion de l'e-cigarette utilise la notion "d'innocuité». L'aérosol est une «vapeur», ses utilisateurs sont des "vapers", couleurs vives, arômes douceâtres, liquides sans nicotine et designs attractifs séduisent les jeunes prêts à expérimenter. Le Surgeon General constate en 2016 que son utilisation dépasse chez les jeunes de 18-24 l'utilisation de tabac des plus que 25 ans [18]. Elle est liée à la consommation de tabac à fumer. Leventhal étudia des jeunes de 14 ans qui avant l'étude n'avaient jamais fumé du tabac [20]. Après une année, ceux qui avaient expérimenté l'e-cigarette fumaient plus que leurs camarades qui n'avaient pas eu affaire à elle. Comme pour d'autres dépendances, les initiés à la nicotine, tôt ou tard, toucheront à la meilleure méthode pour se procurer leur drogue, c.a.d. à la cigarette tabac. Par cela, la promotion de l'e-cigarette est partie du message de l'industrie: «le tabac est discutable, sa consommation est réservée à la décision d'adultes». Ceci parle au goût naturel pour le risque et la transgression de la jeunesse. La décision de notre parlement d'interdire la vente du tabac aux mineurs, mais pas sa publicité, soutient cette stratégie. Illustrant ceci, on trouve parmi les recrues Suisses que les "vapers» se déclarent après 15 mois plus fréquement fumeurs de tabac, en comparaison avec leurs camarade non "vapers» [21].

\section{Conclusions}

Depuis 1900 l'épidémie de tabagisme montre que son étendue dépend plus de la manipulation chimique du produit vendu et de sa promotion commerciale que des effets de la plante elle-même. L'industrie déforma les hypothèses des experts en arguments de vente, dont le contenu ne fut plus corrigé dans la perception publique, comme l'illustrent les exemples de la cigarette à filtre et la cigarette «légère». Le débat «réduction du risque par la cigarette électronique» ressemble à ces démarches perverses. L'industrie réussit ainsi à banaliser la nicotine, la gestuelle de fumer et le tabac, mais aussi à se présenter comme un partenaire de la santé publique. Les marchands citent la "réduction du risque de $95 \%$ » comme prouvée par des études scientifiques. Toutefois ce chiffre repose uniquement sur l'avis d'un groupe d'experts invités à indiquer leur perception subjective de la nocivité par rapport à la cigarette tabac [22]. De même, la commission fédérale de prévention du tabagisme rédigea en 2014 une prise de position [23], dont il a fallu corriger en 2016 les ambivalences [24]. Elles émanaient d'experts tabacologues interrogés par un questionnaire Delphi. Ce procédé reflète typiquement l'opinion de la direction de l'étude, qui n'avait de surcroît pas consulté des experts pédiatres. Les parlementaires sont mis au défi de suivre la CCLAT et d'ancrer dans la LPtab l'interdiction globale de la publicité, de la promotion ainsi que du parrainage pour le tabac et d'y soumettre également les nouveaux produits. L'e-cigarette, utile à un fumeur motivé à cesser son tabagisme, n'a pas besoin de publicité; le conseil professionnel suffit. Enfin, la réduction du nombre de fumeurs dans les pays nordiques et en Australie, prouvent que l'interdiction globale de la publicité est efficace quand elle est appliquée [25].

Crédits

๑) Gawriloff | Dreamstime.com 